however, the treatment decision should be considered in light of the patient's age, comorbidity and wishes regarding post-treatment health status.

Original article Kulkarni GS et al. (2007) Optimal management of high-risk T1G3 bladder cancer: a decision analysis. PLoS Med 4: e284

\section{Stress implicated in the etiology of benign prostatic hyperplasia}

The excessive growth of prostate tissue and increased contractility that occurs in benign prostatic hyperplasia (BPH) causes uncomfortable lower urinary tract symptoms. BPH arises because of subtle dysfunctions in the sympathetic nervous system and the hypothalamic-pituitary-gonadal axis, both of which are highly susceptible to psychological factors.

Ullrich et al. examined the relationship between psychological stress and BPH using measured responses to a standardized laboratory stress test in a group of 83 men who had been diagnosed with BPH. All were asked to perform a standardized laboratory stress task; changes in blood pressure, testosterone and cortisol levels were measured during the task. The degree of physiological response was then examined for potential correlation with the severity of BPH disease. This was assessed by measurements of prostate volume, postvoid residual urine volume, urine flow rate, selfreported lower urinary tract symptoms, and impact and bother scores.

Analysis revealed that increased diastolic blood pressure reactivity was associated with more-severe BPH symptoms, including a greater transition zone volume, a greater total prostate gland volume, greater postvoid residual urine volume, more-severe lower urinary tract symptoms and greater impact scores. Increased cortisol reactivity was associated with elevated bother and impact scores.

The authors conclude that higher physiological responses in a standardized laboratory stress test in men with $\mathrm{BPH}$ are associated with more-severe $\mathrm{BPH}$ disease. They suggest that this is further evidence that stress could be involved in the etiology of prostatic hyperplasia.

Original article Ullrich PM et al. (2007) Physiologic reactivity to a laboratory stress task among men with benign prostatic hyperplasia. Urology 70: 487-492

\section{Early treatment of urinary tract infection prevents renal involvement}

Early treatment of pyelonephritis has been suggested to limit the extent of subsequent renal damage, although this effect has not been demonstrated in prospective clinical trials and remains controversial. In a recent study, Doganis and colleagues investigated the correlation of renal scintigraphy findings with the time interval between the onset of fever and the initiation of antibiotic therapy in 278 infants (aged $\leq 12$ months) hospitalized with a first urinary tract infection.

Antibiotic therapy was initiated a median of 2 days after the onset of fever (range 1-8 days). Overall, renal inflammatory changes were seen in $57 \%$ of the infants, with bilateral defects observed in $13 \%$ of these. The prevalence of renal defects increased with increasing time between fever onset and treatment initiation; defects were seen in $41 \%$ of infants treated within the first day of infection, and $59 \%, 68 \%$ and $75 \%$ of the infants treated on days two, three, and four or later, respectively ( $P<0.0005$ for trend). In total, 76 patients had an abnormal scan in the acute phase of infection and underwent renal scintigraphy a second time (at a median of 6.5 months after fever onset). In these infants the frequency of renal scarring did not differ between those treated in the first 24 hours, and those treated later (46\% vs 54\%), suggesting that while early antibiotic therapy can reduce the likelihood of renal involvement, it does not reduce the likelihood of developing renal scarring once acute pyelonephritis has occurred.

Original article Doganis D et al. (2007) Does early treatment of urinary tract infection prevent renal damage? Pediatrics 120: e922-e928

\section{Successful treatment of female SUI with autologous myoblast and fibroblast injection}

In female stress urinary incontinence (SUI), resting tone and contractibility of the rhabdosphincter are reduced, leading to incomplete closure of the urethra. Mitterberger and coworkers have evaluated the therapeutic potential of autologous myoblasts and fibroblasts, which have previously shown efficacy in animal 